\title{
Study of the degree of deuteration on the microhardness of deuterated potassium dihydrogen phosphate crystals
}

\author{
BAOAN LIU ${ }^{1}$, SHAOTAO SUN ${ }^{2}$, BO WANG ${ }^{1}$, XUN SUN $^{1, *}$, ZHENGPING WANG $^{1}$ and \\ XINGUANG XU ${ }^{1}$ \\ ${ }^{1}$ State Key Laboratory of Crystal Materials, Shandong University, Jinan 250100, PR China \\ ${ }^{2}$ Administration Center, Shandong Academy of Information and Communication Technology, Jinan 250101, PR China
}

MS received 7 October 2013; accepted 1 May 2015

\begin{abstract}
A series of deuterated potassium dihydrogen phosphate (DKDP) crystals with different degrees of deuteration are grown from aqueous solution by the point-seed technique. The microhardness of (100), (001) and so-called 'tripler' faces for these DKDP crystals were measured. Initially the hardness number of (001) face for each crystal increases with the increase of the applied load until it reaches $25 \mathrm{~g}$. With further increase in load, the hardness number decreases gradually. The hardness numbers decline with the increase in deuterium content. These composition dependences are expected since the bond strength is weakened by the substitution of deuterium for hydrogen. The hydrogen bond is considered to play the key role in effecting the crystal's hardness. The visible hardness anisotropy of the different faces is attributed to the inhomogeneous distribution of the oxygen-hydrogen bond on these faces.
\end{abstract}

Keywords. DKDP crystal; deuteration level; hardness.

\section{Introduction}

Potassium dihydrogen phosphate $\mathrm{KH}_{2} \mathrm{PO}_{4}(\mathrm{KDP})$ and its deuterated analog $\mathrm{K}\left(\mathrm{H}_{1-x} \mathrm{D}_{x}\right)_{2} \mathrm{PO}_{4}$ (DKDP) are important optical crystal materials used for electro-optic switches and frequency conversion crystals in high-power large-aperture laser systems. ${ }^{1}$ DKDP crystals are usually used for the third harmonic generation crystals, known as the tripler. ${ }^{2}$ The main problem of these crystals is the relatively low threshold for the formation of damage at light intensities well below the intrinsic laser-induced breakdown threshold. The formation of laser-induced damage is mainly due to ultrafast local material melting, resulting in plastic deformation and fracture of the surrounding material. ${ }^{3,4}$ Therefore, the investigation for the deformation and fracture behaviour of these crystals is helpful to recognize the features of laser-induced damage in these crystals.

As the hardness properties are basically related to the crystal structure of the material, microhardness studies have been applied to understa nd the plasticity of the crystals. ${ }^{5-8}$ The mechanical properties of single-crystal KDP have previously been studied using Vickers microhardness testing. ${ }^{9-12}$ The hardness of $\mathrm{KD}_{2 x} \mathrm{H}_{2(1-x)} \mathrm{PO}_{4}$ (with quoted $x$ values of 0.00 , $0.18,0.50$ and 0.95 ) has been studied using microindentation by Shaskol'skaya and co-workers ${ }^{9}$ who showed that the room-temperature Vickers hardness decreases from 1.48 to $1.28 \mathrm{GPa}$ with the increase in (bulk) deuteration $x$ from 0 to 0.95 . Kucheyev et $a l^{11}$ reported the deformation behaviour

\footnotetext{
*Author for correspondence (sunxun@sdu.edu.cn)
}

of $\mathrm{KD}_{2 x} \mathrm{H}_{2(1-x)} \mathrm{PO}_{4}$ crystals $(x=0.00 .3$, and 0.6 ) by nanoindentation with a $1 \mu \mathrm{m}$ radius spherical indenter. Moreover, within experimental error, they found that the deformation behaviour is independent of the deuterium content and different between (100) face and (001) face. The apparent controversy between these results of hardness on the effect of deuteration showed that the former may be affected by variations of defects and impurities influenced by growth conditions and the latter was attributed to the effect of tensile stress associated with deuterium-hydrogen exchange. ${ }^{12}$ Hence it is necessary to investigate the effect of deuteration on hardness of DKDP crystal without these disturbances.

In this paper, the microhardness of (100), (001) and the tripler faces for DKDP crystals with different deuteration levels which were grown under the same conditions by the point-seed technique was addressed. The effect of deuterium level on these results is analysed. The indentation size effect is also given and discussed.

\section{Experimental}

\subsection{Crystal growth}

A series of DKDP crystals were grown from deuterated aqueous solution in standard $5000 \mathrm{ml}$ glass crystallizers by the rapidly temperature reduction method. The deuterated aqueous solution was made by dissolving high purity $\mathrm{KH}_{2} \mathrm{PO}_{4}$ raw material into the heavy water by adding double-distilled water with resistivity of $18 \mathrm{M} \Omega \mathrm{cm}^{-1}$ to control the degree of deuteration. The content in mass of the main metallic 
ionic impurities in $\mathrm{KH}_{2} \mathrm{PO}_{4}$ raw material is below $0.5 \mathrm{ppm}$. At the beginning, the highest deuterated DKDP crystal was grown from solution with deuteration level is $90 \%$. Then the remaining solution was used to grow the crystal with relatively low deuteration level by adding KDP raw material and ultra-pure water. The deuteration levels of the other solutions were $80,70,60,50,40,30,17$ and $0 \%$. The procedure of crystal growth has been described elsewhere. ${ }^{13}$ All crystals were grown from the same saturation temperature and were uniform in growth rate. The effective distribution coefficient of deuterium is described by the following exponential equation, $k_{\text {eff }}=0.68 \exp (0.00382 M)$, where $k_{\text {eff }}$ is defined here as the mole $\%$ ratio of deuterium in the crystal to deuterium in the solution, $M$ is the mole $\%$ deuterium in the solution. ${ }^{14}$ There are no visible macroscopic defects in these crystals. A typical picture of the crystal visualized along the [010] direction is shown in figure 1a.

\subsection{Mechanical hardness measurements}

Samples for mechanical hardness measurement were cut from nine $\mathrm{K}\left(\mathrm{H}_{1-x} \mathrm{D}_{x}\right)_{2} \mathrm{PO}_{4}$ crystals with mole deuterium content $x$ ranging from 0 to 0.85 . They were, respectively, cut in two orientations: (1) $z$ cut with (001) face and (100) face; (2) type II frequency conversion orientation with the optical axis tipped at $59^{\circ}$ from the $z$-axis, otherwise known as tripler cut with the tripler face. Then the surfaces were orientated by X-ray diffraction with accuracy in $\pm 7^{\circ}$ and fine polished using a conventional manual polishing method with nonaqueous slurries. All polished surfaces were in a high level of optical surface finish. After that, the samples were rapidly stored in a sealed glass vessel with silica gel in it.

The microhardness parameters were determined using a digital Vickers microhardness tester (HXS-1000A). The indentor is in the form of a square pyramid, the opposite faces of which make an angle of $136^{\circ}$ with one another.
The indentation time was fixed as $1 \mathrm{~s}$ for all the samples. For static tests, loads varying from 5 to $100 \mathrm{~g}$, were applied on (100), (001) and the tripler faces of the crystal. Loads were applied gently. Moreover, one diagonal of the diamond indenter is along the [100] direction in the (001) or the tripler faces, and the [010] direction in the (100) face. The distance between any two consecutive indentations was kept at more than thrice the diagonal length of the indentation mark. Thus surface effects were independent of each other. For each load, several impressions were made and then the diagonals $(d)$ were averaged. At least five impressions for each load $(P)$ were recorded. The Vickers hardness numbers $\left(H_{\mathrm{v}}\right)$ were calculated using the formula:

$$
H_{\mathrm{v}}=1.8544 P / d^{2},
$$

where $P$ is in $\mathrm{kg}, d$ in $\mathrm{mm}$ and $H_{\mathrm{v}}$ in $\mathrm{kg} \mathrm{mm}^{-2}$.

After the measurement, the indentation marks were observed by a Carl-Zeiss microscope (Axio Lab. A1).

\section{Results and discussion}

\subsection{Nature of cracks}

Figure 1 shows some examples of the formation of cracks on the surfaces of the crystal with deuterium concentration $x=0.32$. The cracks appearing around the indentations can be classified into two groups: (1) radical cracks originating from the corners of indentation and (2) series of small lateral cracks initiating from the indentation edges and developing in the subsurface layer. At low loads, indentation deformation occurs in the form of dislocation rosette as a result of mobility of dislocations. There are no visible chips around the indentation when the load is below $25 \mathrm{~g}$. While the load is $25 \mathrm{~g}$, radial cracks start from the corner of the indentation. Moreover, a slight deformation region appears
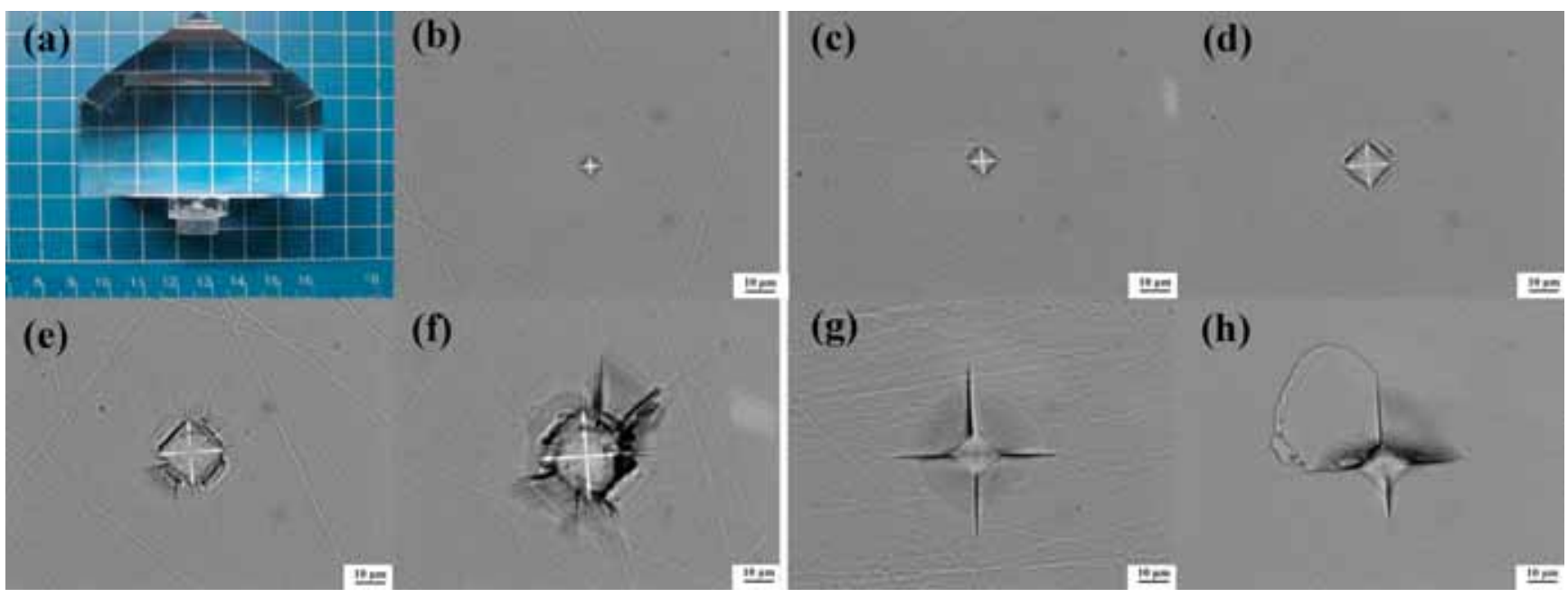

Figure 1. (a) Photograph of the as-grown $\mathrm{K}\left(\mathrm{H}_{1-x} \mathrm{D}_{x}\right)_{2} \mathrm{PO}_{4}$ crystal with composition $x=0.32$. Indentation marks of the crystal with composition $x=0.32$ for (b) $5 \mathrm{~g}$, (c) $10 \mathrm{~g}$, (d) $25 \mathrm{~g}$, (e) $50 \mathrm{~g}$, (f) $100 \mathrm{~g}$ load on (001) face; (g) $25 \mathrm{~g}$ load on (100) face and (h) $25 \mathrm{~g}$ load on the tripler face. 
around the indentation. With further increase in load, lateral cracks occur beneath indentations and spread out laterally on a plane closely parallel to the specimen surface. At higher applied load, rosette-type deformation occurs around indentations.

In addition, from figure 1 it can be seen that the rhombic indentations on (100) face and the tripler face are obviously larger than that on (001) face. As it is quite evident in this figure, these indentations have led to straight and radial cracks with respect to the (001) face. Particularly, scallopshaped fragment appears between two neighboring cracks of the indentation on the tripler face. It can be explained that half of the oxygen-hydrogen $(\mathrm{O}-\mathrm{H})$ bond in KDP structure tilts with the tripler face.

\subsection{Indentation size effect (ISE)}

From figure 2, it can be seen that the microhardness of these crystals depends on the applied load. Initially the hardness number of (001) face for each crystal increases with the increase of the applied load until it reaches $25 \mathrm{~g}$. Indeed a machining hardening effect will appear at the thin layer of the surface machined from incision to grinding and polishing, which could lead to the higher hardness of the thin surface. Under the thin surface, the damage layer is more severe, including the dislocation, plastic deformation, forming of subgrains and residual stress consisting of thermal stress, mechanical stress and strain stress. ${ }^{12,15,16}$ Moreover, the formation of $\mathrm{D} / \mathrm{H}$ exchange layer on the surfaces of DKDP crystals creates a strained depletion layer at the first $\sim 0.5$-m-thick near-surface of the DKDP crystal. ${ }^{17}$ With the increase of deformation energy, the hardness values at the thin surface are higher. With the increasing load, the indentor passes through the depletion layer and the damage layer. The effect of subsurface disturbed layer will diminish. With further increase in load, the hardness number decreases gradually. This phenomenon, known as the ISE, usually

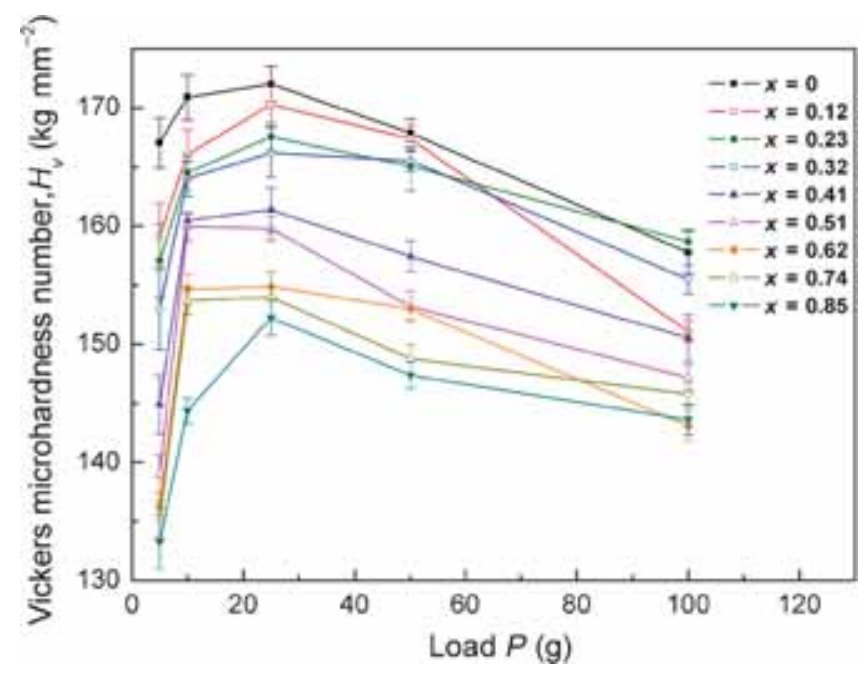

Figure 2. Variation of Vickers hardness number $H_{\mathrm{v}}$ with applied load $P$ on (001) face for different deuterated concentration $x$. involves a decrease in the apparent microhardness with increasing applied test load. The hardness number reaches its maximum value at the load of $25 \mathrm{~g}$. As the indentations made at this load appear without visible cracks, the hardness of the other faces will be tested at $25 \mathrm{~g}$.

Here the ISE is described using the simple Mayer' law. ${ }^{18}$ The expression regarding load and the size of the indentation is given as

$$
P=k_{1} d^{n}
$$

where $k_{1}$ is a constant, $n$ the work hardening coefficient and other symbols have their usual meanings. From the above relation, it is clear that $H_{\mathrm{v}}$ increases with $P$ if $n>2$ and will decrease with $P$ if $n<2$. Experimentally, we can determine $n$ for several compositions from a straight line graph of log $P$ vs. $\log d$. After least squares-fitting, the plot of $\log P$ with $\log d$ fitting data before cracking is given in figure 3. The values of $n$ for all compositions lie between 1.96 (for $x=0$ ) and 2.05 (for $x=0.85$ ) and in between for other intermediate compositions. Because the variation of the coefficients for these crystals is slight, just two plots are given here to avoid superposition. Generally speaking, the values of $n$ will lie between 1 and 1.6 for hard materials and more than that for softer ones. The variation of the work hardening coefficient with concentration $x$ is shown in figure 4, which shows that the value of $n$ increases with the increase of deuterium content $(x)$. However, the whole mixed system lies in the softmaterial category. Note that the ISE is usually related to the deviation of the $n$-value from two, for $n$ is equal to two in the absence of an ISE. In this measurement the small deviation of $n$-value from two indicates that the ISE observed is negligible. The experimental errors resulting from the limitations of the resolution of the objective lens and the sensitivity of the load cell ${ }^{19}$ may conceal the increasing tendency in measured hardness as load increases when the $n$-value is greater than two. From figure 4 it is also found that the value of $n$ for

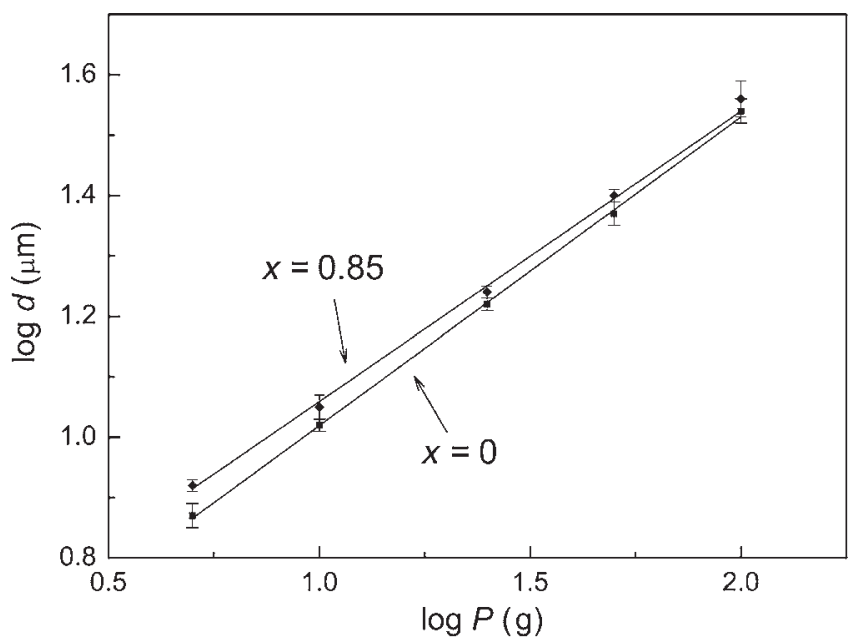

Figure 3. Log-log Meyer plot for $\mathrm{K}\left(\mathrm{H}_{1-x} \mathrm{D}_{x}\right)_{2} \mathrm{PO}_{4}$ crystals $(x=0$ and 0.85$)$. 


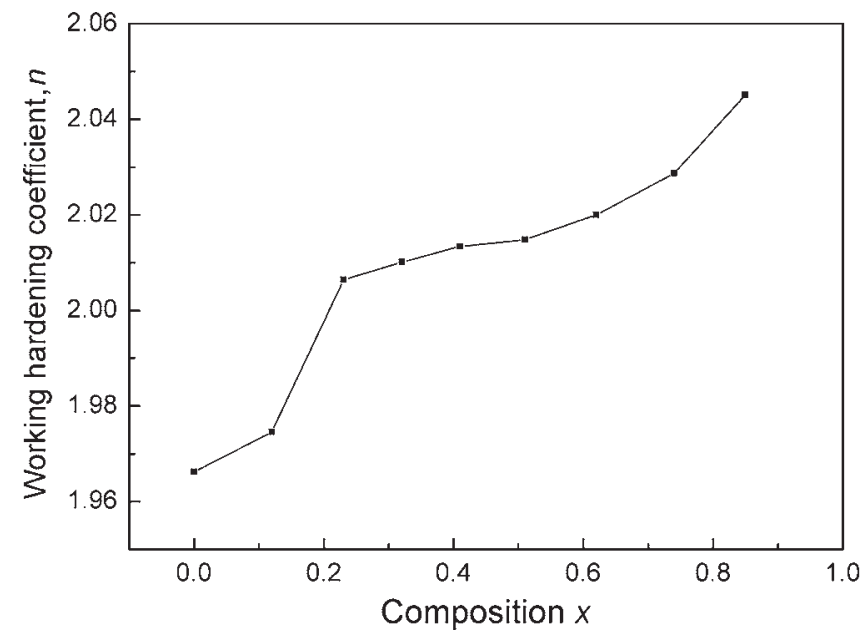

Figure 4. Variation of the work hardening index $n$ with molar concentration $x$.

$\mathrm{KH}_{2} \mathrm{PO}_{4}$ is lower than that of $\mathrm{K}\left(\mathrm{H}_{0.15} \mathrm{D}_{0.85}\right)_{2} \mathrm{PO}_{4}$. This indicates that the strength of crystal decreases with the increase of deuterium content, which agrees well with the experimental values of $H_{\mathrm{v}}$. For brittle materials, ISE is caused by the generation of cracks around indentation. ${ }^{20}$

\subsection{Dependence of hardness on deuteration level}

The composition dependence of hardness for different faces is given in figure 5. It shows that the hardness numbers of these faces decline with the increase of deuterium content. These results are expected since the hydrogen $(\mathrm{H})$ bond strength is weakened by the substitution of deuterium for hydrogen. ${ }^{13,21}$ The hardness values of (001) face are larger than that of other two faces. The hardness values of (100) face are similar to the tripler face when the deuterium content is below 0.51 . With the increasing of deuterium content, the hardness of the tripler face decreased more rapidly with compared to the (100) face.

The observed mechanical anisotropy on different faces of these DKDP crystals can be explained by analysing chemical bonds in KDP structure. The KDP crystal belongs to the acentric tetragonal point group $\mathrm{I} \mid 42 d$ at room temperature. As we know, the structure of $\mathrm{KH}_{2} \mathrm{PO}_{4}$ can be described as $\mathrm{PO}_{4}$ tetrahedral joined by four hydrogen bonds to neighboring tetrahedral, in such a way that each $\mathrm{PO}_{4}$ tetrahedron has two hydrogen atoms associated with it. ${ }^{22}$ This results in an open hydrogen-bonded network that can accommodate the $\mathrm{K}^{+}$ions necessary to preserve charge neutrality. All constituent chemical bonds of KDP crystal may be categorized into three types by the direction and distribution in the lattice. The first type are $\mathrm{K}-\mathrm{O}$ ionic bonds $(2.897 \AA$ ), which are practically parallel to $c$-axis and are drawn in black dotted lines in figure $6 .{ }^{23}$ The second type are also denoted as $\mathrm{K}-\mathrm{O}$ ionic bonds ( $2.824 \AA)$, its directions generally run parallel to (001) face and are illustrated in black dotted lines. The last kind of chemical bonds in KDP crystal are particularly denoted as

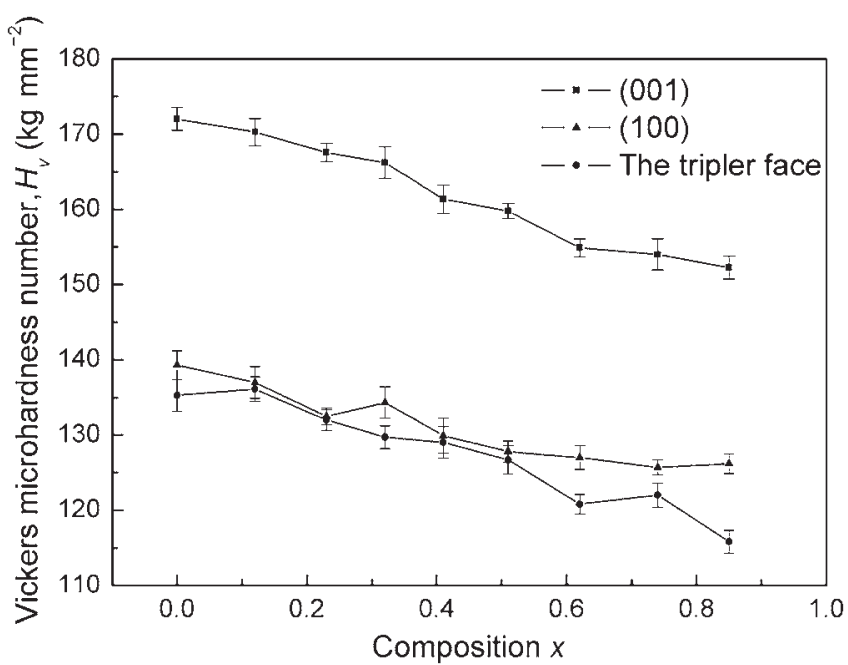

Figure 5. Variation of Vickers hardness number $H_{\mathrm{V}}$ with the composition $x$ for different measurement planes (load $P=25 \mathrm{~g}$ ).

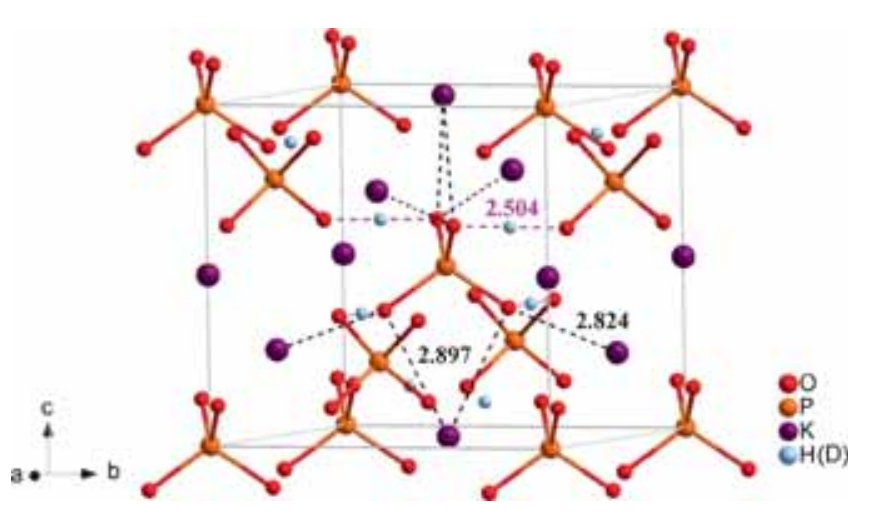

Figure 6. Schematic side view of the tetragonal unit cell of DKDP crystal. The dotted lines represent various chemical bonds formed by body centred $\mathrm{PO}_{4}$ group with adjacent atoms.

hydrogen bonds occurring between two adjacent $\mathrm{PO}_{4}$ groups via $\mathrm{O}-\mathrm{H} / \mathrm{O}$ bonds, which are perpendicular to $c$-axis and represented by pink dotted lines. The hardness of the ideal single crystal is proportional to the bond strengths and to their number in the unit cell of the crystal. ${ }^{24,25}$ Among these bonds, the hydrogen bond has the weakest bond strength. Then it may play the key role in deciding the resistance to permanent deformation or damage of KDP crystal. The number of O-H bond distributed in (001) face is more than that in the (100) face or the tripler face. It is due to the fact that the $\mathrm{O}-\mathrm{H}$ bond is parallel to the [100] or [010] direction. The crystal is easily distorted along this direction, which results in its mechanical fragility. The damage threshold in the direction of the $c$-axis is about two times higher than that of the $a(b)$ axis. ${ }^{4}$

\section{Conclusion}

A series of DKDP crystals with different degrees of deuteration are grown from aqueous solution by point-seed 
technique. The Vickers hardness numbers of (001), (100) and the tripler faces for these crystals are measured with loads varying from 5 to $100 \mathrm{~g}$. The indentation marks of different loads are analysed based on the mechanical property of KDP crystal. The main conclusions are summarized as following.

1. The hardness number for all faces declines with the increase of deuterium content. These composition dependences are expected since the bond strength is weakened by the substitution of deuterium for hydrogen.

2. We consider that the hydrogen bond plays a principle role in effecting crystal's hardness. Due to the fact that the $\mathrm{O}-\mathrm{H}$ bond is parallel to the [100] or [010] direction, there appears a visible hardness anisotropy for different faces.

3. The work hardness coefficient $n$ of $\mathrm{K}\left(\mathrm{H}_{1-x} \mathrm{D}_{x}\right)_{2} \mathrm{PO}_{4}$ crystal increases with the increase of deuterium content, which agrees well with the experimental values of $H_{\mathrm{v}}$.

\section{Acknowledgements}

This work was financially supported by the Program for New Century Excellent Talents in University (NCET-10-0526), the National Natural Foundation of China (51202131), the Natural Science Foundation of Shandong Province (ZR2010EM001), the Independent Innovation Foundation of Shandong University (IIFSDU, 2012JC016) and the Graduate Independent Innovation Foundation of Shandong University (GIIFSDU, yzc12073).

\section{References}

1. Zaitseva N and Carman L 2001 Prog. Cryst. Growth Charact. Mater. 431

2. De Yoreo J J, Burnham A K and Whitman P K 2002 Int. Mater. Rev. 47113
3. Carr C W, Feit M D, Johnson M A and Rubenchik A M 2006 Appl. Phys. Lett. 89131901

4. Yoshida H, Jitsuno T, Fujita H, Nakatsuka M, Yoshimura M, Sasaki T and Yoshida K 2000 Appl. Phys. B 70195

5. Desai C C and Rai J L 1983 Bull. Mater. Sci. 5453

6. Jain A, Razdan A K, Kotru P N and Wanklyn B M 1994 J. Mater. Sci. 293847

7. Karan S, Sen Gupta S and Sen Gupta S P 2003 Mater. Sci. Eng. A 357304

8. Lawn B R and Marshall D B 1979 J. Am. Ceram. Soc. 62347

9. Fang T and Lambropoulos J C 2002 J. Am. Ceram. Soc. 85 174

10. Guin C H, Katerich M D, Savinkov A I and Shaskolskaya M P 1980 Krist. Tech. 15479

11. Kucheyev S O, Siekhaus W J, Land T A and Demos S G 2004 Appl. Phys. Lett. 842274

12. Lu C, Gao H, Wang J, Teng X and Wang B 2010 Mater. Manuf. Process. 25740

13. Liu B, Yin X, Sun X, Xia M, Ji S, Xu X and Zhang J 2012 J. Appl. Crystallogr. 45439

14. Loiacono G M, Balascio J F, and Osborne W 1974 Appl. Phys. Lett. 24455

15. Gong J, Wu J and Guan Z 1999 J. Eur. Ceram. Soc. 192625

16. Li H and Bradt R C 1993 J. Mater. Sci. 28917

17. Kucheyev S O, Felter T E, Siekhaus W J, Nelson A J and Hamza A V 2004 Appl. Phys. Lett. 841344

18. Meyer M A 1951 Some aspects of the hardness of metals Ph.D. thesis (Delft)

19. Mason W, Johnson P F and Varner J R 1991 J. Mater. Sci. 26 6576

20. Sangwal K 2009 Cryst. Res. Tech. 441019

21. Kucheyev S O, Bostedt C, Van Buuren T, Willey T M, Land T A, Terminello J L, Felter T E, Hamza A V, Demos S G and Nelson A J 2004 Phys. Rev. B 70245106

22. Slater J C 1941 J. Chem. Phys. 916

23. Xu D, Xue D and Ratajczak H 2005 J. Mol. Struct. 74037

24. Gao F, He J, Wu E, Liu S, Yu D, Li D, Zhang S and Tian Y 2003 Phys. Rev. Lett. 91015502

25. Simunek A and Vackar J 2006 Phys. Rev. Lett. 96085501 